\title{
Inducing Microscale Structural Order in Phage Nanofilament Hydrogels via Macromolecular Crowding with Globular Proteins
}

\author{
Azadeh Peivandi, ${ }^{a^{*}}$ Kyle Jackson, ${ }^{a^{*}}$ Lei Tian, ${ }^{a}$ Leon He, ${ }^{a}$ Ahmad Mahmood, ${ }^{b}$ Cécile Fradin, ${ }^{b}$ Zeinab \\ Hosseinidoust ${ }^{a, c \dagger}$
}

${ }^{\text {a }}$ Department of Chemical Engineering, McMaster University, Hamilton, Ontario, Canada

${ }^{\mathrm{b}}$ Department of Physics and Astronomy, McMaster University, Hamilton, Ontario, Canada

${ }^{\mathrm{c}}$ Michael DeGroote Institute for Infectious Disease Research, McMaster University, Hamilton, Ontario, Canada

* Equally contributing first authors

${ }^{\dagger}$ Corresponding Author. Phone: (905) 525-9140; email: doust@mcmaster.ca

\begin{abstract}
Biological hydrogels play important physiological roles in the body. These hydrogels often contain ordered subdomains that provide mechanical toughness and other tissue-specific functionality. Filamentous bacteriophages are nanofilaments with a high aspect ratio that can self-assemble into liquid crystalline domains that could be designed to mimic ordered biological hydrogels and can thus find application in biomedical engineering. We have previously reported hydrogels of pure crosslinked liquid crystalline filamentous phage formed at very high concentrations exhibiting a tightly packed microstructure and high stiffness. In this work, we report a method for inducing selfassembly of filamentous phage into liquid crystalline hydrogels at concentrations that are several orders of magnitude below that of lyotropic liquid crystal formation, thus creating structural order, but a less densely packed hydrogel. Hybrid hydrogels of M13 phage and bovine serum albumin $(0.25 \mathrm{w} / \mathrm{v} \%)$ were formed and shown to adsorb up to $16 \times$ its weight in water. Neither component gelled on its own at the low concentrations used, suggesting synergistic action between the two components in forming the hydrogel. The hybrid hydrogels exhibited repetitive self-healing under physiological conditions and at room temperature, autofluorescence in three channels, and antibacterial activity towards Escherichia coli host cells. Furthermore, the hybrid hydrogels exhibited more than $2 \times$ higher ability to pack water compared to BSA-only hydrogels and $2 \times$ higher flexibility (lower compression modulus) compared to tightly packed M13-only hydrogels, suggesting that our method could be used to create hydrogels with tunable mechanical properties through the addition of globular proteins, while maintaining structural order at the microscale. KEYWORDS: bottom-up assembly, liquid crystal, biorecognition, M13, self-assembly
\end{abstract}




\section{Introduction}

Natural polymers have long garnered interest as building blocks for biomaterials. Natural polymers such as cellulose, ${ }^{1}$ starch, ${ }^{2}$ collagen, ${ }^{3}$ silk, ${ }^{4}$ and chitosan ${ }^{5}$ have been reported to form hydrogels via physical or chemical crosslinking. These polymers are biodegradable and biocompatible by nature and they do not release toxic materials upon degradation. As a special class of natural polymers, proteins and peptides offer several functional groups that make them amenable to a range of chemical reactions, allowing for chemical crosslinking, loading of different drugs, and chemistries enabling trigger-responsive behaviour. ${ }^{6}$ However, proteins/peptides have outstanding limitations as building blocks for biomaterials, namely the possibility of denaturation in the gelation process and the limited bioactivity offered by the protein. Each protein/peptide can offer only a single biorecognition ability, if any, limiting the utility of protein/peptide hydrogels as multifunctional bioactive material. Both limitations can be overcome using viral nanoparticles, specifically bacteriophages.

Bacteriophages, or phages for short, are viruses that infect bacteria. Phages offer remarkable diversity both in terms of genomic sequence and physical appearance and can be found in many shapes and sizes. ${ }^{7}$ Phages are essentially proteinous nanoparticles that are amenable to all chemistries applicable to proteins, with the additional advantage that the chemistry of the bacteriophage protein coat can further be controlled with atomic precision via genetic engineering, a property that allows for designing made-to-order, multifunctional nanoparticles that can selfpropagate, and thus makes phages superior to synthetic nanoparticles. For biomaterials in general, and hydrogels in particular, specific interaction and biorecognition of select ligands (organic and inorganic) is a highly desired property.

Filamentous phages of Escherichia coli $(f 1, f d$ and M13) are proteinous nanofilaments with a high aspect ratio and circular DNA genome. ${ }^{8}$ Filamentous phages offer properties that present them as unique building blocks for the bottom up synthesis of advanced material. M13 phage, in particular, has been extensively explored for its capacity as a bionanoparticle for applications ranging from design of tissue engineering scaffolds to energy storage devices. ${ }^{9}$ At high concentrations, filamentous phages self-organize into liquid crystalline phases. ${ }^{10-12}$ Previously, we reported hydrogels made entirely from M13 filamentous phage, self-organized into lyotropic liquid crystals prior to crosslinking, and showed that these hydrogels have a range of advanced properties. ${ }^{13}$ These hydrogels, however, required very high concentrations of M13 to form lyotropic liquid crystals 
prior to crosslinking, leading to a densely packed structure at the microscale which led to low flexibility.

Liquid crystal phase transition in rod shaped particles has been modeled to be induced through addition of non-adsorbing polymers or micro/nanoparticles through a phenomenon known as macromolecular crowding or depletion interactions. ${ }^{14,15}$ Building on these theoretical models, we designed a hybrid hydrogel with M13 filamentous phage and the small globular protein BSA (bovine serum albumin). Adding a very low concentration of BSA to the M13 suspension achieved structural order at the microscale by inducing liquid crystal formation through macromolecular crowding. The M13 suspension used were at concentrations up to 50,000-fold lower than the onset of lyotropic liquid crystal formation. BSA was added at concentrations so low that it would not gel if mixed with a crosslinker, and neither BSA nor the M13 phage would show birefringence or form a hydrogel alone. We further investigated the swelling and mechanical properties of these hybrid hydrogels and demonstrated that our hybrid hydrogels exhibit all the advanced properties of a dense M13-only hydrogel, namely self-healing, autofluorescence, and biological activity towards host bacterial cells, but exhibit higher flexibility.

\section{Results and Discussion}

\section{Physical characterization of hybrid M13 hydrogels}

M13 phage at $2 \times 10^{12} \mathrm{PFU} / \mathrm{mL}$, purified using aqueous two-phase system method to remove proteins, peptides, and nucleic acids, was crosslinked with different BSA concentrations using glutaraldehyde as a crosslinker. The crosslinking reaction occurs between amine groups in lysine residues of M13 phage ( 340) and BSA (30-35) protein with two aldehyde groups in glutaraldehyde. ${ }^{16}$ BSA concentrations as low as $0.25 \mathrm{w} / \mathrm{v} \%$ resulted in strong gelation, whereas lower BSA concentrations lead to a degree of transparency change in the mixture, suggesting presence of crosslinked regions, but the mixture would flow freely at room temperature (Figure 1A). At the concentrations used, M13 would not gel without any BSA. It is noteworthy that BSA alone in Millipore water did not gel at concentrations below $3 \mathrm{w} / \mathrm{v} \%$ (Figure S1). The fact that neither of the two components comprising the hybrid hydrogels were able to form a gel alone suggests a degree of synergy between M13 and BSA in forming the hybrid hydrogel. We further measured the gelation time using the tube inversion method (Figure S2). As expected, gelation time increased exponentially with decreasing BSA concentration, ranging from a few minutes to $24 \mathrm{hrs}$ for the lowest BSA concentration. During the process of gelation, glutaraldehyde, an 
aggressive carbonyl $(-\mathrm{CHO})$ reagent, condenses amine groups of lysine residues on the phage coat protein as well as BSA protein via Mannich reaction or reductive amination.

In order to analyze the liquid crystalline structures that are formed by M13 phages, polarized light microscopy was used to observe birefringence. Birefringence is an optical property of a material having a refractive index that depends on the polarization and propagation direction of light. ${ }^{17}$ Polarized light microscopes resemble that of conventional light microscopes apart from the addition of two crossed polarizers; one below the sample stage just above the condenser lens (called the polarizer) and the other just above the objective before the tube lens (called the analyzer). ${ }^{18}$ Birefringence is defined as the maximum difference between the minimum and maximum indices of refraction. When inbound light passes through the polarizer, it becomes linearly polarized. If the material is birefringent, then this linearly polarized light can become elliptically polarized, acquiring a component in the direction perpendicular to that of the polarizer which will be allowed to pass through the analyzer. The amount of light that is detected after the analyzer is then dependent on the relative angle of the sample and the crossed polarizers. This is what we observed for suspensions of M13 at concentration of $10^{12} \mathrm{PFU} / \mathrm{mL}$ when even a very low concentration of BSA $(0.05 \%)$ was added (Figure 1B, C). If the sample is not birefringent, then the light will not be able to pass through the analyzer resulting in a dark field. Birefringence is absent in samples of M13+BSA at M13 concentrations below $10^{10} \mathrm{PFU} / \mathrm{mL}$ or BSA concentrations below $0.05 \%$ (Figure S3A). The evaluation of the strength of the birefringent properties in Figure S3A was based on empirically-derived evidence. We compared the samples to a pure solution of highly concentrated M13 bacteriophage solution that had undergone liquid crystal formation. A strong birefringent sample was defined as one for which an amount of light similar to that of the pure M13 bacteriophage solution was detected in the polarization microscope. In comparison, a weak birefringent sample was defined as one which demonstrated reduced light transmission relative to the pure M13 bacteriophage solution. Figures S3B-F show representative polarized micrographs of M13-BSA mixtures at different concentrations during the crosslinking process. All subsequent results are reported for a concentration of $1-2 \times 10^{12} \mathrm{PFU} / \mathrm{mL}$, because of the reasonable gelation time, unless otherwise stated. 

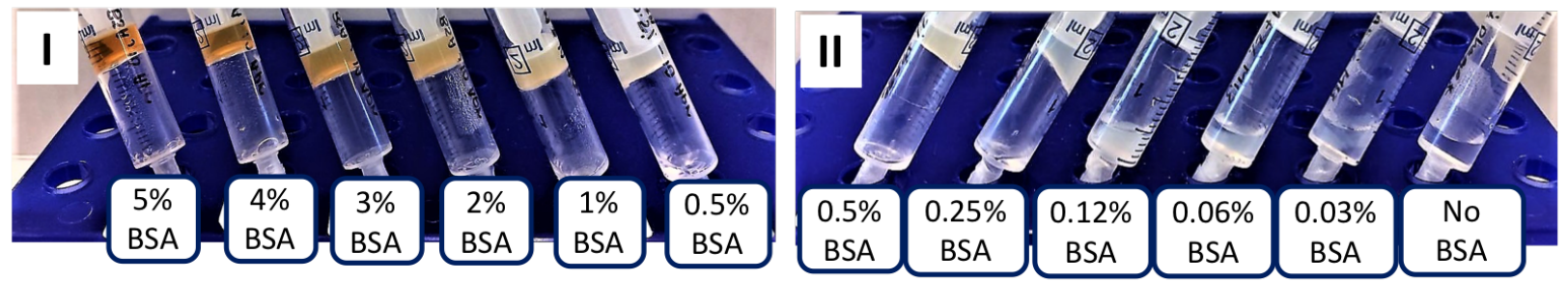

(B)
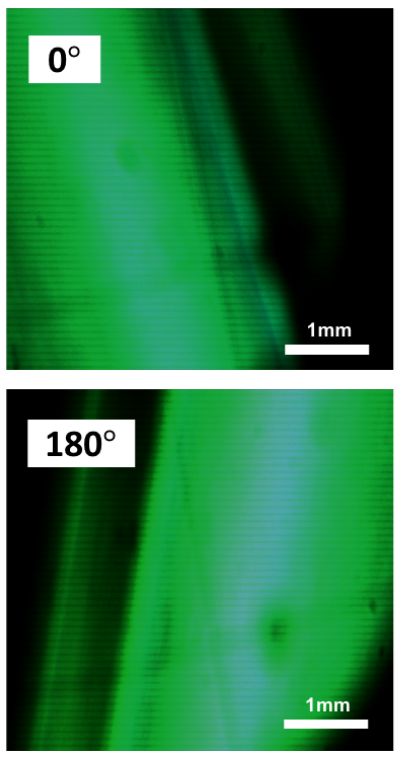
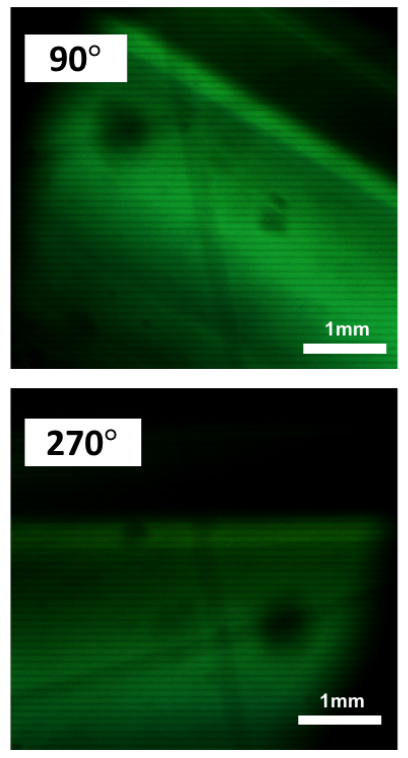

(C)
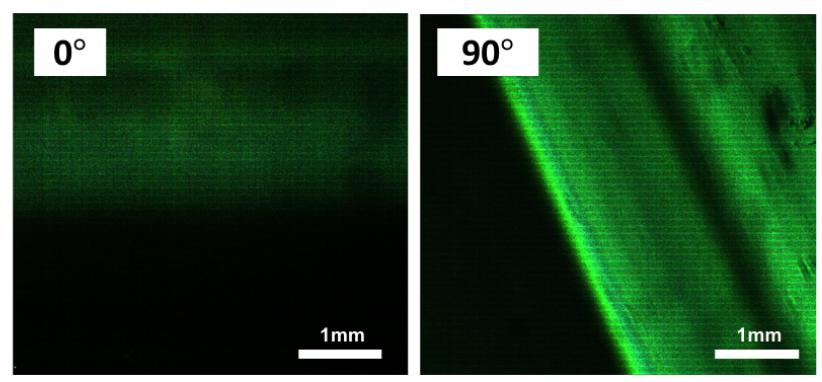

$180^{\circ}$

Figure 1- (A) Images of hydrogels; I, II. pictures of glutaraldehyde-crosslinked hybrid M13-BSA hydrogels made with $\sim 10^{12}$ PFU/mL of M13 and different BSA concentrations. (B) Polarized optical micrographs of M13-BSA hybrid hydrogels with $\sim 10^{12}$ PFU/mL of M13 and $1 \%$ BSA, at different relative angles between sample and crossed polarizers $\left(0,90,180\right.$, and $\left.270^{\circ}\right)$. (C) Polarized optical micrographs of M13-BSA hybrid hydrogels with $10^{12}$ PFU/mL of M13 and $0.05 \%$ $B S A$, at different relative angles between sample and crossed polarizers $\left(0,90,180\right.$, and $\left.270^{\circ}\right)$. All gels shown were crosslinked with $1 \%$ glutaraldehyde.

When comparing hydrogels of BSA alone (at $3 \mathrm{w} / \mathrm{v} \%$, the limit of BSA solo gelation) and hybrid M13-BSA hydrogels ( $10^{12} \mathrm{PFU} / \mathrm{mL}$ M13, 3\% BSA), hybrid hydrogels showed a significantly higher swelling ratio $(\sim 14 \times$ their dry weight) compared to hydrogels with BSA alone ( $\sim 4 \times$ their dry weight). Decreasing BSA concentration from 3 to $1 \%$ slightly increased swelling ratio to $\sim 15 \times$ their dry weight, suggesting a possible change in pore structure in the presence of BSA (Figure 2A). Swelling at temperatures other than room temperature $\left(\sim 25^{\circ} \mathrm{C}\right)$ showed a slight decrease (from 
$13 \times$ to $10 \times$ ) with increasing swelling temperature to $37^{\circ} \mathrm{C}$, but no effect at lower temperatures (Figure 2B).
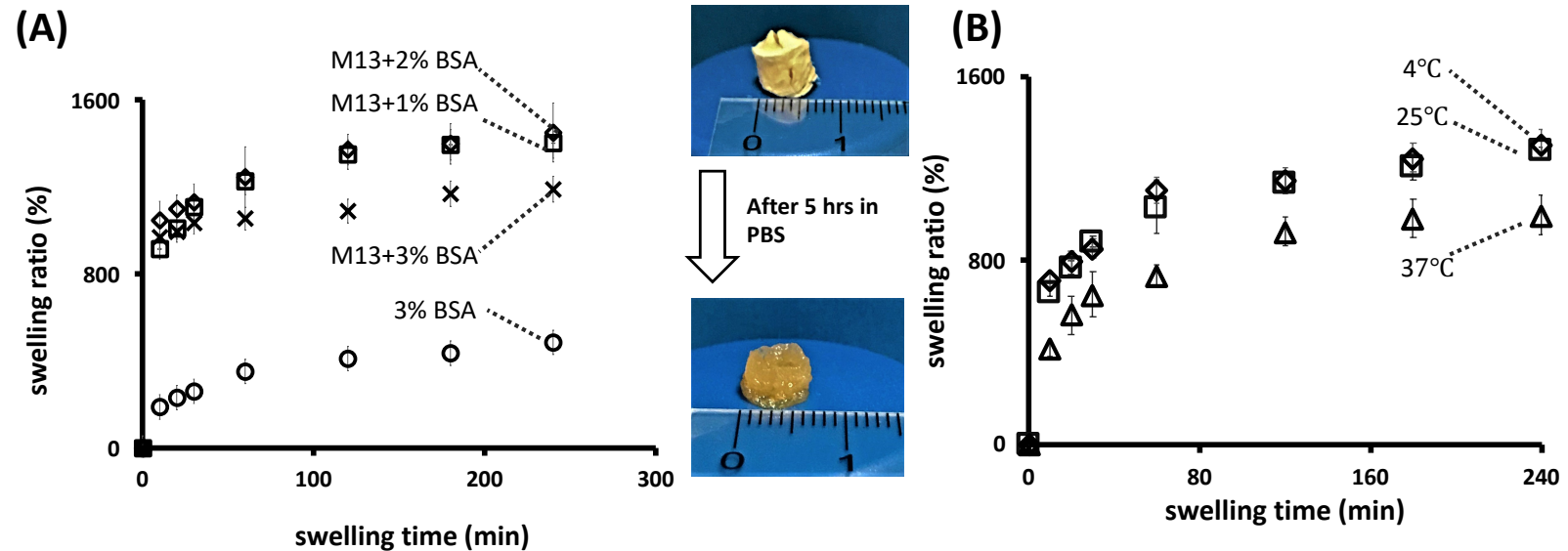

Figure 2- (A) Swelling ratio for hybrid M13-BSA hydrogels with different concentration of BSA. Image shows picture of a freeze-dried hybrid M13-BSA(1\%) hydrogel after 5 hours swelling at $25^{\circ} \mathrm{C}$. (B) Effect of temperature on swelling ratio of the hybrid M13-BSA hydrogels.

\section{Mechanical and chemical characterization of hybrid M13 hydrogels}

SEM images of the hybrid M13-BSA hydrogels (1\% BSA, crosslinked with 1(w/v)\% glutaraldehyde, critical point dried) revealed microporous structure with a fine pore network (Figure 3A, B). For comparison, Figures 3C, D show the structure of a freeze-dried hydrogel, which, as expected, show a larger pore size than critical point dried hydrogels, likely as a results of ice crystal formation. Furthermore, comparing the pore structure of hybrid M13-BSA (3\%) hydrogels (Figure 3E, F) with BSA hydrogels with the same concentration of BSA (Figure 3G, H) revealed the hybrid M13-BSA hydrogels to have a finer pore structure, with shapes that are distinctly different, partially explaining the difference observed in swelling ratio.

Addition of BSA, however, appeared to have strengthened the hydrogels, based on the stress-strain response curves in Figure 4A. The increase of BSA percentage from 1 to 3\% significantly increased the amount of stress the hybrid hydrogels could sustain. The compression test results in a nonlinear curve, making the calculation of a global modulus of compression difficult, therefore we calculated the initial slope of the stress-strain curve below 5\% strain. The experimental observation showed that there was no breaking point of the hydrogels under pressure up to $25 \mathrm{~N}$, although they were pressed with dimensional changes under this force. The hybrid M13-BSA 
hydrogels with $1 \%$ BSA exhibited a compression modulus of $0.2 \mathrm{KPa}$ that increased to $1.6 \mathrm{KPa}$ for M13- BSA hydrogels with 3\% BSA, a value which is at least half that of the previously reported liquid crystalline, M13 only hydrogels, ${ }^{13}$ suggesting an increased in flexibility of the hybrid hydrogels over the close-packed, pure M13 hydrogels. It is noteworthy that should mechanical strength be desired, these hydrogels can be strengthened by adding natural nanoparticles known for their mechanical strengths, such as cellulose nanocrystals. Many polymeric hydrogels also show a soft behavior, which depending on the application, could be an advantage or a disadvantage. The increased flexibility of our hybrid hydrogels could, for example, be advantageous for coating on wound dressing material.
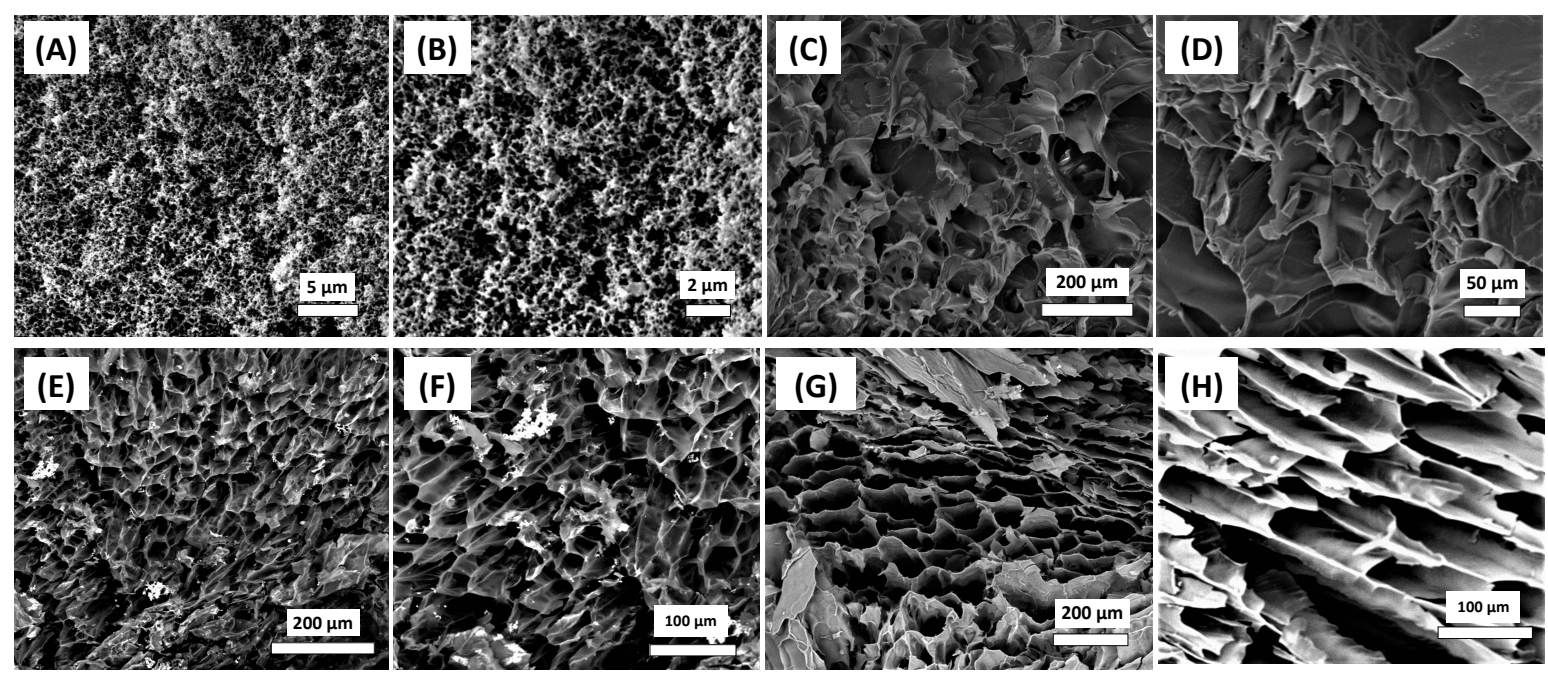

Figure 3- Scanning electron micrographs of $(\boldsymbol{A}, \boldsymbol{B})$ hybrid $M 13+1 \%$ BSA hydrogel, critical point dried; (C,D) hybrid M13+1\% BSA hydrogel, freeze dried; $(\boldsymbol{E}, \boldsymbol{F})$ hybrid M13+3\% BSA hydrogel; $(\boldsymbol{G}, \boldsymbol{H}) 3 \%$ BSA hydrogel.

We previously reported repetitive self-healing of M13 hydrogels in the presence of $\mathrm{CaCl}_{2} \cdot{ }^{13} \mathrm{We}$ observed the hybrid M13-BSA hydrogels to exhibit similar behavior at room temperature (Figure 4C). When the hydrogels, cut with a razor blade, were incubated at room temperature in the presence of $2.6 \mathrm{mM} \mathrm{CaCl}_{2}$ (extracellular physiological levels) the hydrogels started to show signs of healing within hours, completely fusing back together into an intact hydrogel within 24 hrs. Interestingly, the healed hydrogel showed a comparable albeit lower compression modulus to the intact hydrogels ( $\sim 0.5 \mathrm{vs} \sim 0.8 \mathrm{KPa}$ for the intact hybrid hydrogels with $2 \% \mathrm{BSA}$ ) (Figure 4D), 
suggesting an almost biomimetic degree of self-healing with minimal compromise to mechanical properties. It has been reported that BSA can be physically crosslinked in the presence of $\mathrm{Ca}^{2+},{ }^{19}$ which may explain the self-healing property of our hybrid M13-BSA hydrogels. However, for BSA to be physically crosslinked with $\mathrm{Ca}^{2+}$, the protein must be first heated to thermally unfold the native BSA protein and form fibrillar BSA aggregates, then gelation is induced by adding fresh native BSA into the solution in the presence of $\mathrm{Ca}^{2+}$ at room temperature. ${ }^{19}$ There are no reports of BSA being physically crosslinked with $\mathrm{Ca}^{2+}$ at room temperature without an initial step of heatinduced aggregation. The origin of self-healing must be explored further, however the observation that our hybrid M13-BSA hydrogels heal under physiological conditions suggests that these hydrogels can be expected to exhibit self-healing similar to biological tissue where the healing processes are autonomously triggered after the damage.
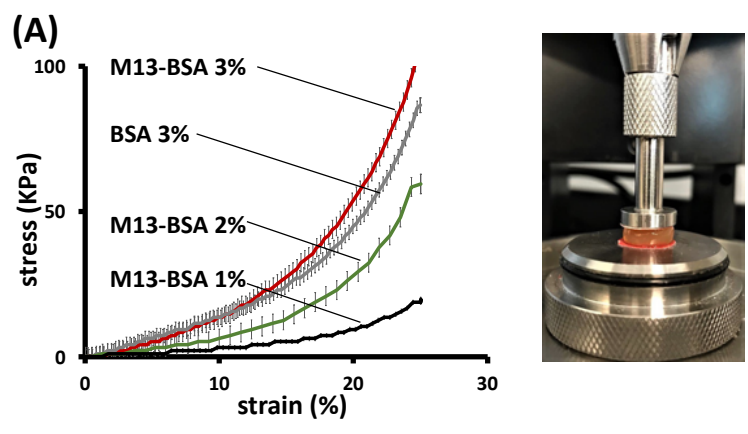

(B)

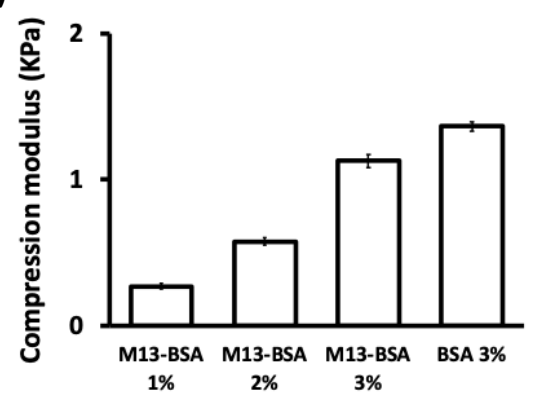

(C)

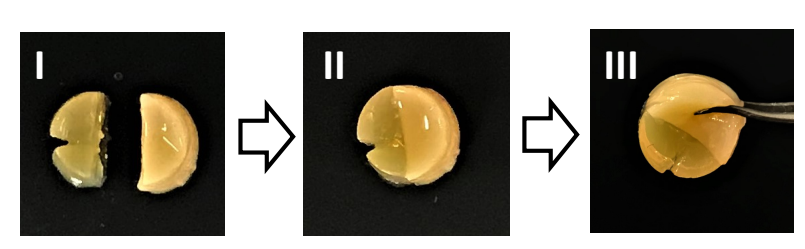

(D)

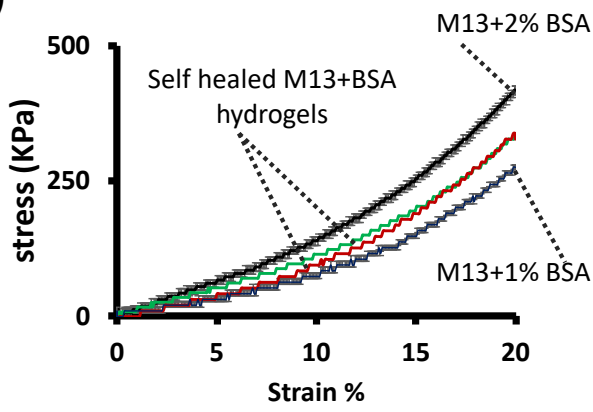

Figure 4- (A) Stress-strain response for hybrid M13-BSA and BSA hydrogels; image shows a hybrid M13-BSA hydrogel under compression load. (B) Compression modulus for hybrid M13$B S A$ and BSA hydrogels. (C) Self-healing of two different M13-BSA hybrid hydrogels made with different BSA concentrations. I. Two different hydrogels, one nicked for clarity, II. The self-healed gel after incubation in $2.4 \mathrm{mM} \mathrm{CaCl}$, III. demonstrating the stability of the healed region to withstand the weight of the gel. (D) compression behavior for M13 hydrogel before and after selfhealing in different BSA concentrations. 
The hybrid M13-BSA hydrogels and the BSA only hydrogels, both exhibited autofluorescence. However, while BSA only hydrogels showed autofluorescence at red and green wavelengths (Figure S4A), hybrid M13-BSA hydrogels auto fluoresced at green, red, and blue wavelengths (Figure S4B). Autofluorescence with the optical filter set ex/em $=358 / 461 \mathrm{~nm}$, appeared to be an added property of M13 hydrogels, since it was also observed in our previous report with M13 only hydrogels. BSA solution (Figure S5A), M13 phage suspensions (Figure S5B), glutaraldehyde (Figure S5C) did not show any autofluorescence when imaged with the same set of filters. Glutaraldehyde is known to react with proteins and peptides to generate visible to near-IR emitters such as ethylenediamine and secondary amines. ${ }^{20}$ This suggests that the observed autofluorescence could be a result of electronic transitions such as $\pi-\pi^{*}$ transition of $\mathrm{C}=\mathrm{C}$ bond in glutaraldehyde and $\mathrm{n}-\pi^{*}$ transition of $\mathrm{C}=\mathrm{N}$ bonds in the Schiff's base (result of reaction between $\alpha, \beta$-unsaturated aldehyde polymers in glutaraldehyde and M13 surface proteins). ${ }^{21}$ The type of protein that reacts with glutaraldehyde, size of the protein or peptide and $\mathrm{pH}$ of the reaction affects the by-products of the reaction and thus the nature of this autofluorescence, ${ }^{20}$ offering a versatile platform for preparing bioactive hydrogels for imaging applications.

To investigate possible production of fluorescent chemicals, FTIR spectra of the hybrid hydrogel was analysed in comparison to the M13 suspension and glutaraldehyde. Both the phage suspension and the hydrogel showed the same amide I peaks at $\sim 1660 \mathrm{~cm}^{-1}$, demonstrating amide bond in protein capsid of M13 phage (Figure S4C). In addition, the hydrogel exhibited amide II ( $\sim 1570$ $\left.\mathrm{cm}^{-1}\right)$, which is also seen for BSA suspension, and amide III bonds $\left(\sim 1280 \mathrm{~cm}^{-1}\right)$. There are multiple new absorption peaks (aside from those shared with glutaraldehyde, BSA solution, and M13 suspension) that suggest new functional groups/new compounds formed. The UV-vis spectrum further showed that the hybrid M13-BSA hydrogel at $450 \mathrm{~nm}$, whereas the phage suspension and BSA solution had a sharp absorption peaks at $\lambda=280 \mathrm{~nm}$, a typical absorption peak for proteins (Figure S4D). We further confirmed the biological activity of the hybrid M13-BSA hydrogels by probing the ability of the M13 phage crosslinked inside the hydrogel to infect their bacterial host, to confirm that the addition of BSA does not in any way interfere with M13 phage infectivity. We incubated the hydrogels with the $E$. coli host for $5 \mathrm{hrs}$ at $37^{\circ} \mathrm{C}$; the culture showed a relatively high titer of phage suggesting the M13 phage inside the hydrogel was still biologically active and able to infect its host bacteria and propagate itself (Figure S5D). To rule out the possibility of M13 leaking out of the hydrogels, we compared the results for freshly prepared hydrogels with hydrogels 
that were washed in Millipore water (10 water changes, $25 \mathrm{~mL}$ each). The washed hydrogel resulted in the same level of propagated phage as the non-washed hydrogel, when incubated with host bacteria. A $50 \mathrm{KDa}$ protein on tip of the phage fiber must bind to the bacterial cell to initiate the insertion of the M13 genome into the bacterial cell and start the infection and M13 propagation cycle. ${ }^{7}$ This means that as long as the binding proteins are active, even if M13 filaments are not available to freely diffuse through the environment and bind to their host cells, they can still infect host bacteria, thus maintaining their much desired biological activity in the hydrogel. M13 bioactivity in the hydrogel matrix offers possibilities for the preparation of bioactive hydrogels that offer multiple different types of biorecognition, engineered through recombinant DNA techniques and/or phage display.

\section{Conclusions}

We report the synergistic action of M13 filamentous phage and BSA, a small globular protein leading to the formation of a liquid crystalline domains through macromolecular crowding. Neither the M13 phage, nor BSA can from hydrogels at the concentrations used in this work when reacted with $1 \%$ glutaraldehyde. We showed previously that the formation of self-organized domains at very high M13 concentrations was critical for the formation M13 hydrogels. In the current report, however, we managed to form M13 hydrogels at concentrations 50,000× lower than the onset of formation of smectic phase, only by adding low concentrations of BSA protein. The Presence of M13 phage in the hybrid hydrogels significantly increased the swelling ratio of the M13-BSA hydrogels over BSA-only hydrogels $(>3 \times)$, which we hypothesize is because of the change in pore structure between hybrid BSA-M13 hydrogels and BSA-only hydrogels, as observed in electron micrographs. Presence of BSA, however, worked to decrease the compression modulus of the hybrid M13-BSA hydrogels over pure M13 hydrogels. This indicates a compromise between ability to pack water and the mechanical strength of the hydrogel, which must be accounted for when designing hybrid M13-BSA hydrogels based on the final application. Our hybrid hydrogels exhibit all the advanced characteristics of pure M13 hydrogels that make them attractive for biomedical applications, namely autofluorescence in three wavelengths, self-healing under physiological conditions with minimal loss of mechanical strength and antimicrobial activity towards their host bacterial cells. Our report highlights the utility of hybrid M13-BSA hydrogels for development of bioactive multifunctional material as well as the importance of further mechanistic investigations into the nature of the self-healing in the presence of different proteins 
of biological relevance, which could open avenues for the development of stimulus-responsive biomaterials with M13 phage.

\section{Materials and Methods}

\section{Bacteria and phage culture methods}

Escherichia coli K12 ER2738 (New England Biolabs Ltd.), genotype: F'proA $\mathrm{A}^{+} \mathrm{B}^{+}$lacl $^{\mathrm{q}}$ $\Delta$ (lacZ)M15 zzf::Tn10(Tet $\left.{ }^{\mathrm{R}}\right) /$ fhuA2 glnV $\Delta$ (lac-proAB) thi-1 $\Delta$ (hsdS-mcrB)5, was used as host for phage M13. Pre-cultures of host were prepared in LB-Miller broth (Fisher Scientific) using a single colony from fresh tetracycline plates (streaked from glycerol stocks) and incubated in a shaking incubator at $37^{\circ} \mathrm{C}$, overnight. The preculture was then diluted 1:100 in $250 \mathrm{~mL}$ of LB broth, to which $10 \mu \mathrm{L}$ of M13 phage stock at $10^{12}$ plaque forming units (PFU) per mL was added. The phage culture was incubated shaking at $37^{\circ} \mathrm{C}$ for $5 \mathrm{hrs}$. The culture was subsequently centrifuged $(7000 \times \mathrm{g}, 15 \mathrm{~min})$ to pellet bacteria. The supernatant, containing phage, was saved and stored at $4^{\circ} \mathrm{C}$.

\section{Phage purification and concentration}

The crude phage stock was purified via aqueous two-phase method, as described by Sambrook. ${ }^{22}$ Briefly, a mixture of $20(\mathrm{w} / \mathrm{v}) \%$ PEG solution and $2.5 \mathrm{M} \mathrm{NaCl}$ solution was added to the crude phage stock with a volumetric ratio of 1:6 and incubated at $4^{\circ} \mathrm{C}$ overnight. Phage was pelleted by centrifugation $\left(5000 \times \mathrm{g}, 45 \mathrm{~min}, 4^{\circ} \mathrm{C}\right)$. The pellet was resuspended in $10 \mathrm{~mL}$ of RO Millipore water (resistivity $=18.2 \mathrm{M} \Omega . \mathrm{cm}$ ) and incubated on a tube roller at $4^{\circ} \mathrm{C}$ for $2 \mathrm{hrs}$. Tubes were subsequently centrifuged $(5000 \times \mathrm{g}, 15 \mathrm{~min})$ to remove any residual bacterial debris. This purification step was repeated twice. The concentration of M13 phage was quantified using the plaque assay method, as described elsewhere. ${ }^{23}$

\section{Preparation of hydrogels}

Different concentrations of M13 phage suspension and BSA solution were prepared by making serial dilution of the purified, concentrated stock in Millipore water. Next, in a $3 \mathrm{~mL}$ disposable syringe, M13 phage was mixed with BSA and 1\% glutaraldehyde and incubated at room temperature for a few minutes to $24 \mathrm{hrs}$. Inversion test was applied to verify gelation. Gelation time was recorded as the time when the crosslinked protein/M13 stops flowing out of inverted tubes at room temperature. The hydrogels were then removed from the syringe and kept submerged in 
Millipore water at room temperature until used for subsequent experiments. Hydrogels were prepared fresh and used within a few hours for all experiments described below.

\section{Swelling test}

Phage hydrogels were flash frozen with liquid nitrogen to decrease ice crystal formation and immediately freeze dried using Labconco lyophilizer. The dried gels were weighed and placed in $20 \mathrm{~mL}$ of $0.1 \mathrm{M} \mathrm{pH}=7.4$ phosphate-buffered saline (PBS) at different temperature $\left(4,25\right.$, and $\left.37^{\circ} \mathrm{C}\right)$. The swollen gels were removed from the solution at the certain time intervals, after gently removing excess water with lint-free tissue paper, the gels were weighed using a Mettler analytical balance (readability $0.1 \mathrm{mg}$ ). Swelling ratio was calculated as follows by using the measured mass of wet gel $\left(\mathrm{m}_{\mathrm{w}}\right)$ and dry gel $\left(\mathrm{m}_{\mathrm{d}}\right)$ : swelling ratio $(\%)=\frac{m_{w}-m_{d}}{m_{d}} \times 100$

\section{Compression test}

Compression tests were carried out at $25^{\circ} \mathrm{C}$ using a Mach-1 Mechanical Tester (Biomomentum Inc, QC) with parallel-plate geometry. Prior to mechanical test, hydrogel discs were prepared with a diameter of $10 \mathrm{~mm}$ and a height of $2-4 \mathrm{~mm}$. Compression testing was performed to $20 \%$ of the sample height at a rate of $0.03 \mathrm{~mm} / \mathrm{s}$. Preload force of $0.01 \mathrm{~N}$ and ramp force of $0.5 \mathrm{~N} / \mathrm{min}$ were applied.

\section{Chemical characterization and spectrometry}

UV-vis spectra of both the phage suspension, hybrid phage hydrogels and BSA solution was recorded using a BioTek plate reader in the range of 200-900 $\mathrm{nm}$. Wavelength scanning was performed between $200 \mathrm{~nm}$ and $900 \mathrm{~nm}$. Attenuated total reflectance infrared (ATR-FTIR) spectra of phage solution and hydrogel were obtained with a Thermo Nicolet IR 560 system, using a ZnSe ATR accessory (Thermo Electron Corporation, PA). Each sample was placed against the ATR element and the spectra were collected in the range of $500-4000 \mathrm{~cm}^{-1}$ using 128 scans at a resolution of $4 \mathrm{~cm}^{-1}$. After acquisition, the IR spectra were baseline corrected for carbon dioxide peak at approximately $2750 \mathrm{~cm}^{-1}$.

\section{Microscopy}

For electron microscopy, the samples were prepared using two different methods, namely freezedrying and critical point drying. For freeze-drying, hydrogels were flash frozen in liquid nitrogen, freeze dried, then freeze fractured. For critical point drying, the hydrated hydrogels were dehydrated using an ethanol gradient and dried using a Leica critical point dryer (EM CPD300). 
The samples were stored in a desiccator and prior to electron microscopy, were coated with a 10 $\mathrm{nm}$ layer of gold. Imaging was performed using at $10 \mathrm{kV}$ a field emission scanning electron microscope (TESCAN VP SEM).

The hydrogels were imaged in hydrated state using a Zeiss inverted fluorescence microscope with three sets of filters, namely ex/em $=358 / 461 \mathrm{~nm}$, ex/em $=470 / 530 \mathrm{~nm}$ and ex $/ \mathrm{em}=595 / 630 \mathrm{~nm}$. The Excitation filter was placed in front of the LED light to excite the hydrogel with the specific wavelength and the emission filter was attached on the camera to record the emission image of the phage hydrogel.

For polarized light microscopy, phage and hydrogel samples were prepared as previously described, suspended in a square glass capillary tube to emulate hydrogel gelation, placed on a glass slide and secured using parafilm. All glass square capillary tubing and slides were first cleaned of particulate matter by subjecting them to a methanol wash and dried with filtered nitrogen gas. The glass slide was then placed on the rotating stage of the polarized light microscope. Samples were visualized using a Nikon light microscope, and two crossed polarizers. Images were captured and analyzed using Motic imaging software. Images were captured every $45^{\circ}$ to display the birefringent properties of the samples.

\section{Self-healing test}

For self-healing experiments, fully hydrated hydrogels were cut into two halves, the halves (from the same or different hydrogels) were put in a same shape mold and kept in contact in the presence of $2.6 \mathrm{mM}$ calcium chloride at room temperature for $24 \mathrm{hrs}$. The healed hydrogel was taken out of the mold and used for either compression test or electron microscopy. Repeated self-healing was performed by cutting a healed hydrogel at a different location and repeating the procedure as described above.

\section{Acknowledgement}

The authors would like to acknowledge funding from Natural Sciences and Engineering Research Council of Canada (NSERC) and the Boris Family Fund for Health Research. We thank Dr. Todd 
Hoare for guidance and access to mechanical testing apparatus. The authors also thank Sina Ghasempour for critical assistance with phage preparations during the earlier stages for this work. 
Supplementary Information

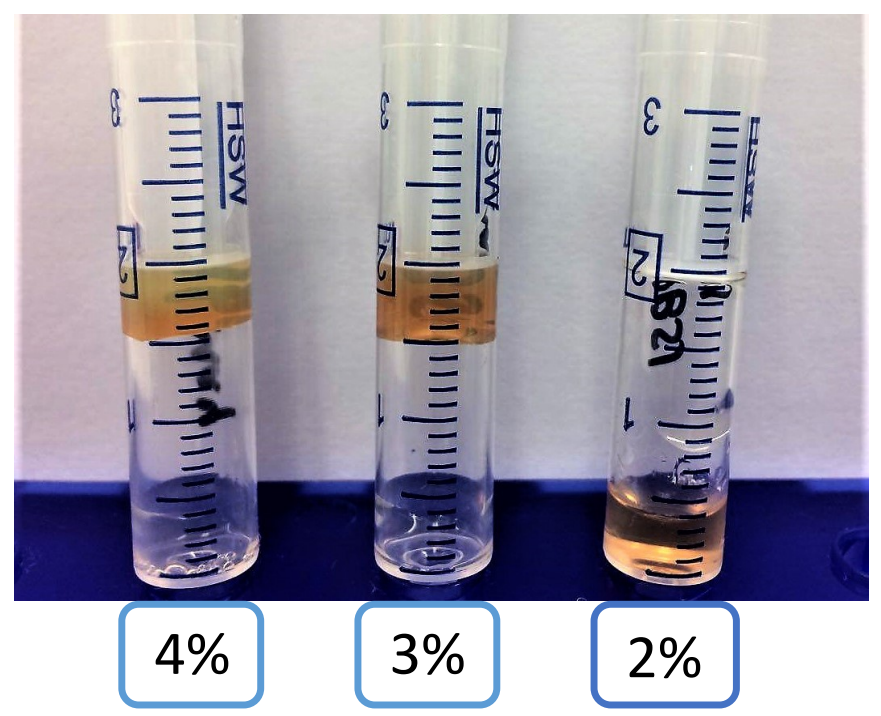

Figure S1-Visual representation of the tube inversion method, confirming gelation for pure BSA and glutaraldehyde.

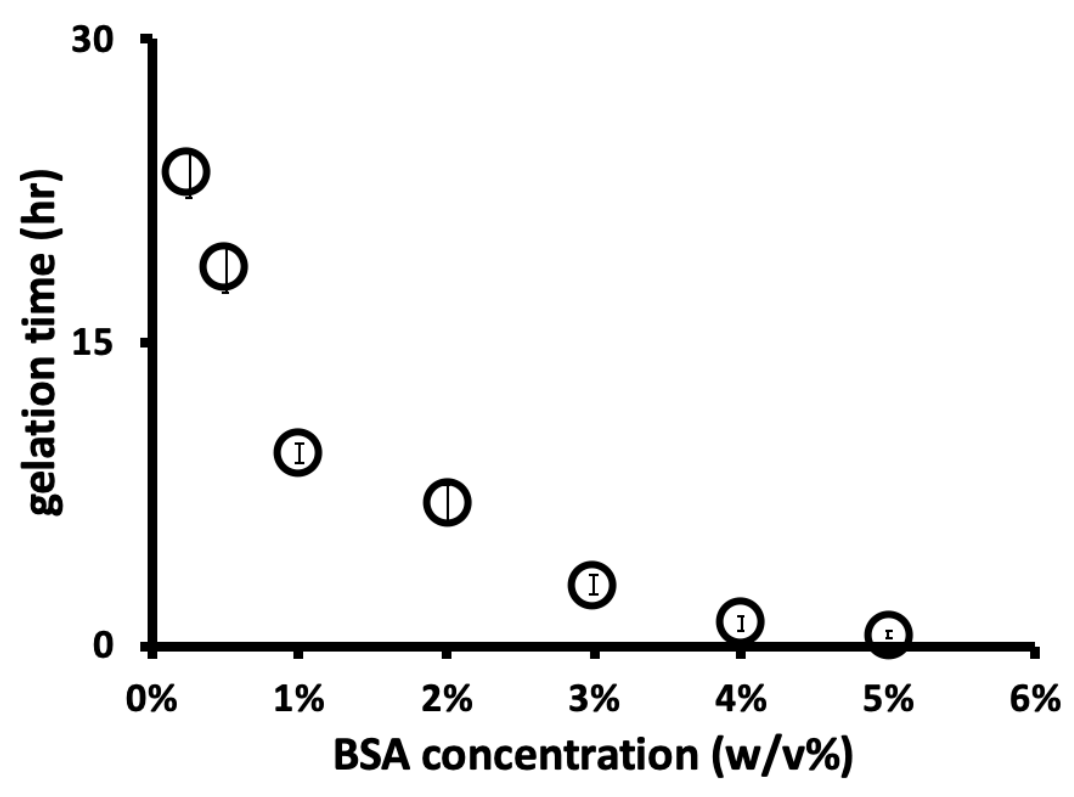

Figure S2- Gelation time for hybrid M13-BSA hydrogels. 


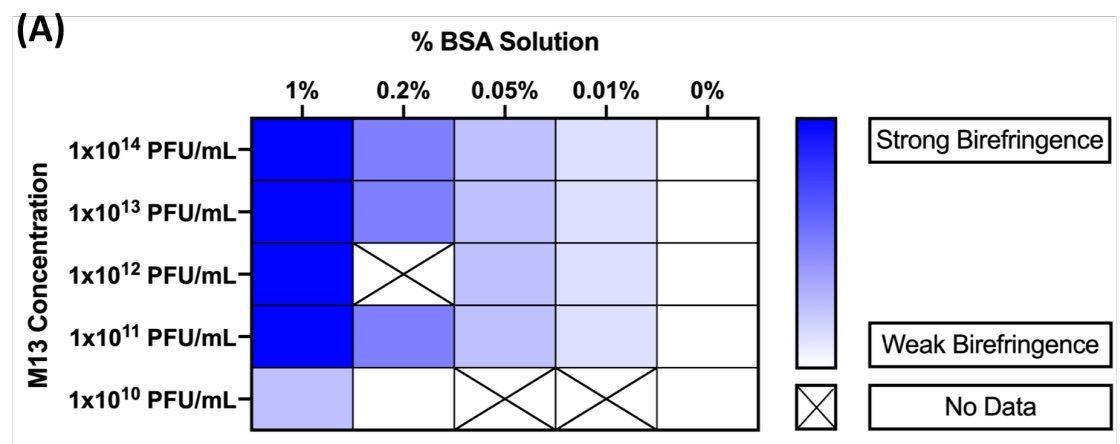

(B) M13: $10^{11} \mathrm{pfu} / \mathrm{mL}$, BSA: $1 \%$

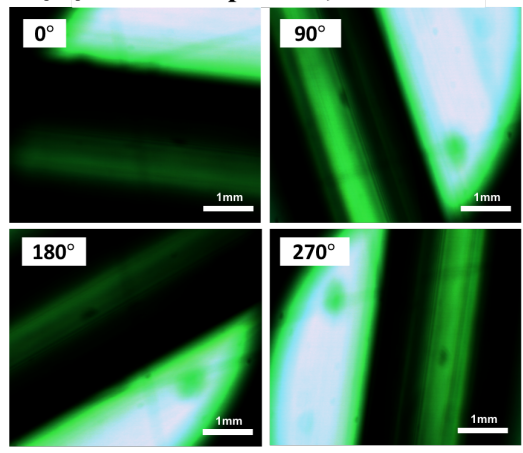

(E) M13: $10^{10} \mathrm{pfu} / \mathrm{mL}$, BSA: $1 \%$

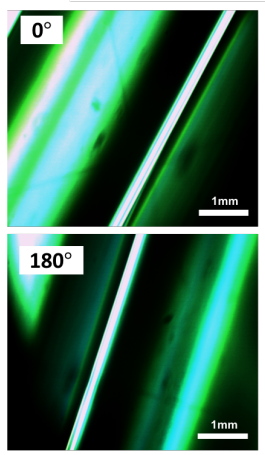

(C) $M 13: 10^{11} \mathrm{pfu} / \mathrm{mL}, \mathrm{BSA}: 0.2 \%$

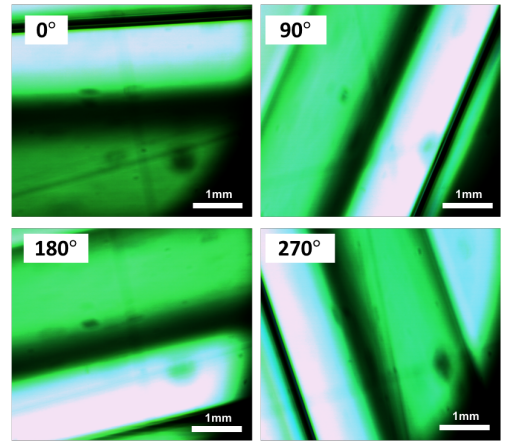

(F) M13: $10^{10} \mathrm{pfu} / \mathrm{mL}, \mathrm{BSA}: 0.2 \%$
(D) M13: $10^{11} \mathrm{pfu} / \mathrm{mL}, \mathrm{BSA}: 0.05 \%$

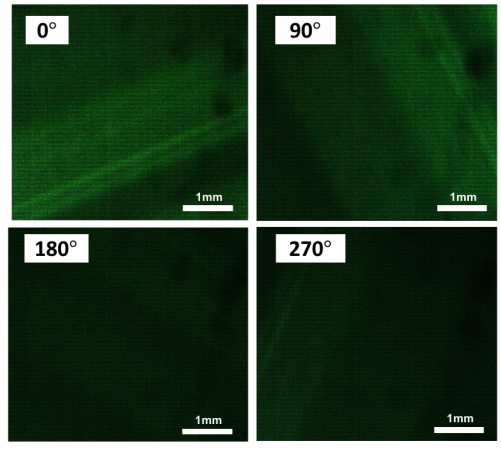

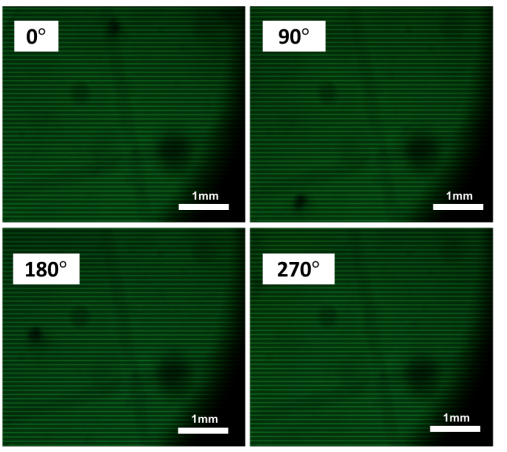

Figure S3-(A) Summary table for birefringence observed in M13-BSA mixtures upon adding the crosslinker. Polarized optical micrographs of M13-BSA hybrid hydrogels at different relative angles of cross polarizers $\left(0,90,180\right.$, and $\left.270^{\circ}\right)$ at concentrations: $(\boldsymbol{B}) \sim 10^{11} \mathrm{PFU} / \mathrm{mL}$ of M13 and 1\% BSA; (C) $\sim 10^{11} \mathrm{PFU} / \mathrm{mL}$ of $\mathrm{M13}$ and $0.2 \% \mathrm{BSA}$; (D) $\sim 10^{11} \mathrm{PFU} / \mathrm{mL}$ of M13 and $0.05 \%$ BSA; (E) $\sim 10^{10} \mathrm{PFU} / \mathrm{mL}$ of $M 13$ and $1 \% \mathrm{BSA}$. (F) $\sim 10^{10} \mathrm{PFU} / \mathrm{mL}$ of M13 and $0.2 \% \mathrm{BSA}$. 
(A)
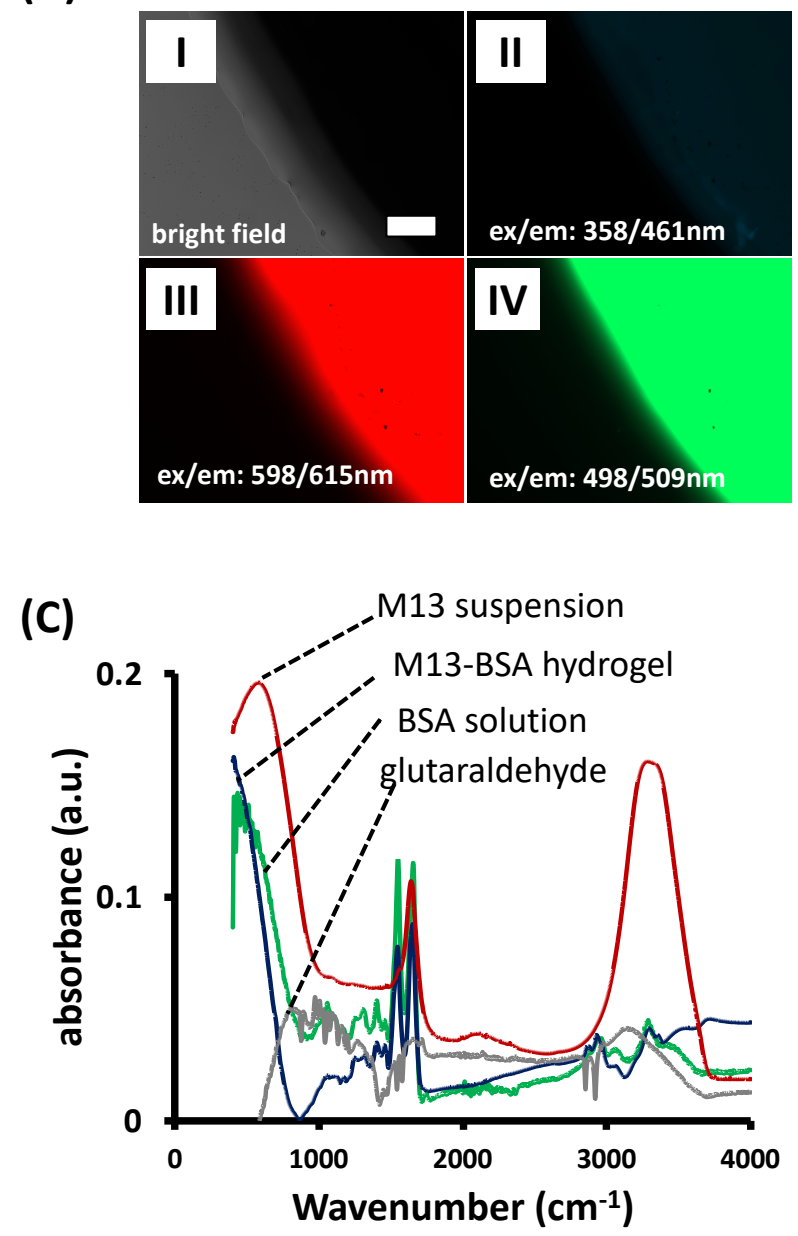

(B)

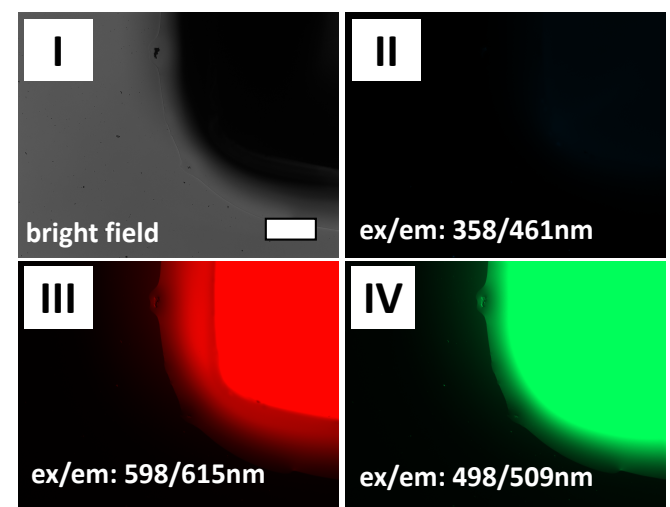

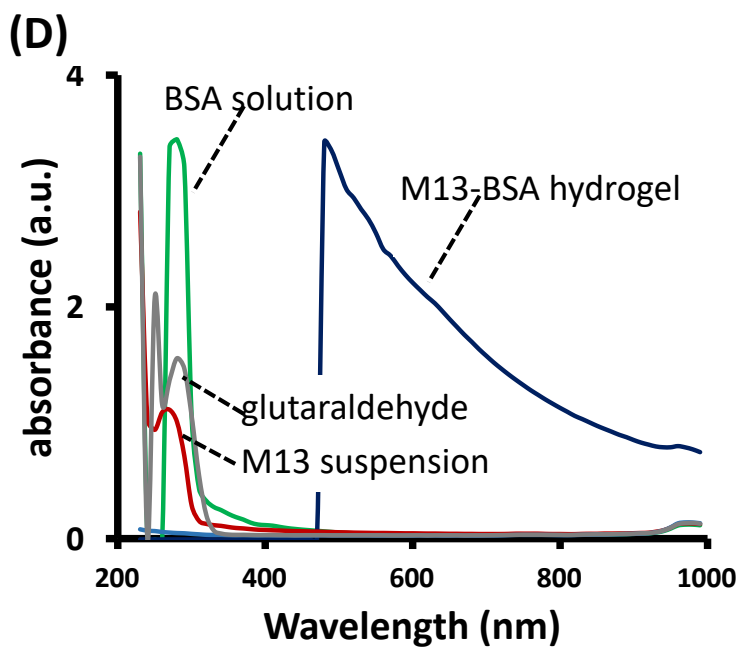

Figure S4- (A) Fluorescence micrographs for hydrogel made with 3\% BSA: I. brightfield, II. hydrogel excited at $358 \mathrm{~nm}$ and imaged using a $\lambda=461 \mathrm{~nm}$ optical filter, III. hydrogel excited at $498 \mathrm{~nm}$ and imaged using a $\lambda=509 \mathrm{~nm}$ optical filter, IV. hydrogel excited at $598 \mathrm{~nm}$ and imaged using a $\lambda=615 \mathrm{~nm}$ optical filter. Scale bar $=200 \mu \mathrm{m}$. (B) Fluorescence micrographs for hydrogel made with $1 \%$ BSA and $2 \times 10^{12}$ PFU/mL M13, using the same filters as part c. The gain was kept constant for all three excitation/emission pairs. Scale bar $=200 \mu \mathrm{m}$. (C) FTIR spectra for M13BSA hydrogel, BSA hydrogel, and BSA solution. (D) UV-Vis spectra of BSA solution, M13 suspension and BSA-M13 hydrogel showing a distinctive peak for the hydrogel. 
(A)

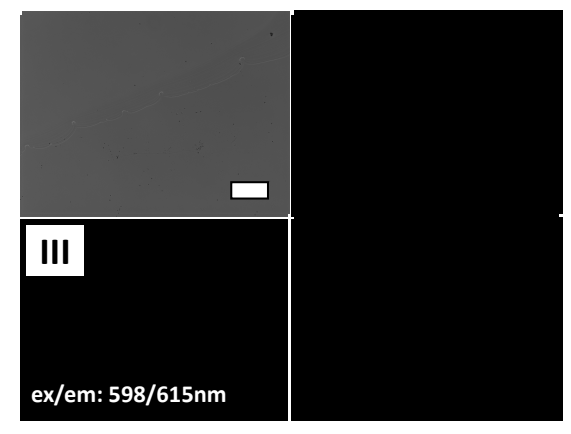

(D)

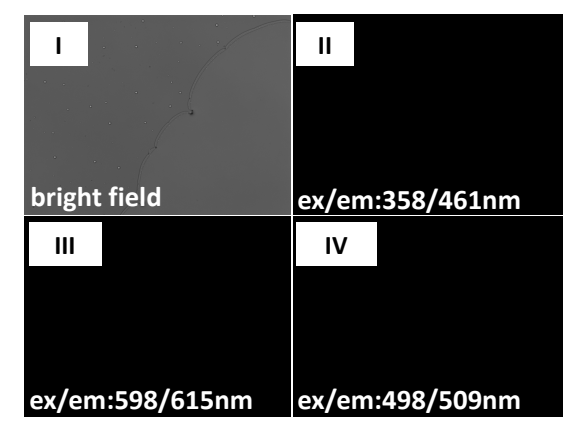

(B)

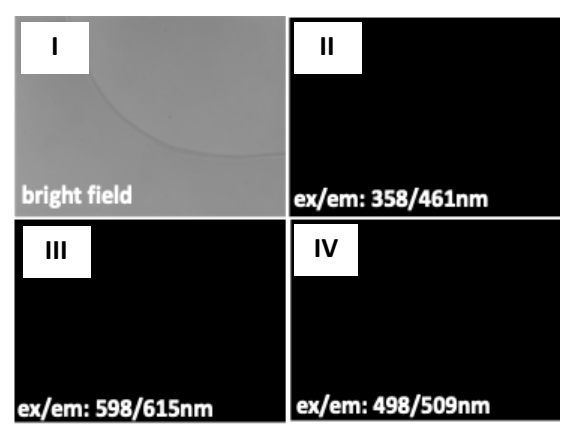

(D)

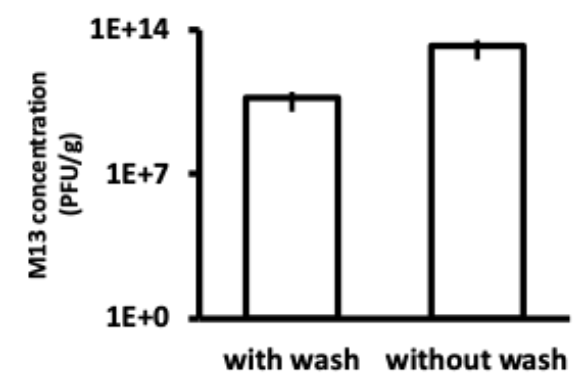

Figure S5- (A) Gelation time for hybrid M13-BSA hydrogels. Brightfield and fluorescence micrographs of (B) BSA solution; (C) $3 \times 10^{12} \mathrm{PFU} / \mathrm{mL}$ M13 phage; (D) glutaraldehyde. 


\section{References}

1. Yousefi, N.; Wong, K. K. W.; Hosseinidoust, Z.; Sørensen, H. O.; Bruns, S.; Zheng, Y.; Tufenkji, $\mathrm{N}$., Hierarchically porous, ultra-strong reduced graphene oxide-cellulose nanocrystal sponges for exceptional adsorption of water contaminants. Nanoscale 2018, 10, (15), 7171-7184.

2. $\quad \mathrm{Xu}, \mathrm{X}$.; Shen, Y.; Wang, W.; Sun, C.; Li, C.; Xiong, Y.; Tu, J., Preparation and in vitro characterization of thermosensitive and mucoadhesive hydrogels for nasal delivery of phenylephrine hydrochloride. Eur. J. Pharm. Biopharm. 2014, 88, (3), 998-1004.

3. Olde Damink, L. H. H.; Dijkstra, P. J.; Van Luyn, M. J. A.; Van Wachem, P. B.; Nieuwenhuis, P.; Feijen, J., Glutaraldehyde as a crosslinking agent for collagen-based biomaterials. J. Mater. Sci. Mater. Med. 1995, 6, (8), 460-472.

4. $\quad$ Kim, U.-J.; Park, J.; Li, C.; Jin, H.-J.; Valluzzi, R.; Kaplan, D. L., Structure and Properties of Silk Hydrogels. Biomacromolecules 2004, 5, (3), 786-792.

5. $\quad$ Nieto-Suárez, M.; López-Quintela, M. A.; Lazzari, M., Preparation and characterization of crosslinked chitosan/gelatin scaffolds by ice segregation induced self-assembly. Carbohydr. Polym. 2016, 141, 175-183.

6. Krall, N.; da Cruz, F. P.; Boutureira, O.; Bernardes, G. J. L., Site-selective protein-modification chemistry for basic biology and drug development. Nat. Chem. 2015, 8, 103.

7. $\quad$ Calendar, R. L., The Bacteriophages. second ed.; Oxford University Press: New York, 2006.

8. Rakonjac, J., Filamentous Bacteriophages: Biology and Applications. 2012.

9. $\quad$ Lee, J. H.; Warner, C. M.; Jin, H.-E.; Barnes, E.; Poda, A. R.; Perkins, E. J.; Lee, S.-W., Production of tunable nanomaterials using hierarchically assembled bacteriophages. Nat. Protocols 2017, 12, (9), 1999-2013.

10. Lapointe, J.; Marvin, D. A., Filamentous Bacterial Viruses VIII. Liquid Crystals of fd. Molecular Crystals and Liquid Crystals 1973, 19, (3-4), 269-278.

11. Dogic, Z.; Fraden, S., Cholesteric Phase in Virus Suspensions. Langmuir 2000, 16, (20), 78207824.

12. Jackson, K.; Peivandi, A.; Fogal, M.; Tian, L.; Hosseinidoust, Z., Filamentous Phages as Building Blocks for Bioactive Hydrogels. ACS Applied Bio Materials 2021.

13. Peivandi, A.; Tian, L.; Mahabir, R.; Hosseinidoust, Z., Hierarchically Structured, Self-Healing, Fluorescent, Bioactive Hydrogels with Self-Organizing Bundles of Phage Nanofilaments. Chem. Mater. 2019, 31, (15), 5442-5449.

14. Dogic, Z.; Fraden, S., Ordered phases of filamentous viruses. Current Opinion in Colloid \& Interface Science 2006, 11, (1), 47-55.

15. Dogic, Z., Filamentous Phages As a Model System in Soft Matter Physics. Frontiers in Microbiology 2016, 7, (1013).

16. Chung, W.-J.; Lee, D.-Y.; Yoo, S. Y., Chemical modulation of M13 bacteriophage and its functional opportunities for nanomedicine. International Journal of Nanomedicine 2014, 9, 5825-5836.

17. Wu, S.-T., Birefringence dispersions of liquid crystals. Physical Review A 1986, 33, (2), 12701274.

18. Newton, R. H.; Haffegee, J. P.; Ho, M. W., Polarized light microscopy of weakly birefringent biological specimens. J. Microsc. 1995, 180, (2), 127-130.

19. Chen, J.; Dong, Q.; Ma, X.; Fan, T.-H.; Lei, Y., Repetitive Biomimetic Self-healing of $\mathrm{Ca}^{2+}-$ Induced Nanocomposite Protein Hydrogels. Sci. Rep. 2016, 6, 30804.

20. Lee, K.; Choi, S.; Yang, C.; Wu, H.-C.; Yu, J., Autofluorescence generation and elimination: a lesson from glutaraldehyde. Chem. Commun. 2013, 49, (29), 3028-3030.

21. Wei, W.; Wang, L.-Y.; Yuan, L.; Wei, Q.; Yang, X.-D.; Su, Z.-G.; Ma, G.-H., Preparation and Application of Novel Microspheres Possessing Autofluorescent Properties. Adv. Funct. Mater. 2007, 17, (16), 3153-3158.

22. Sambrook, J.; Russell, D. W., Molecular cloning: a laboratory manual. 3 ed.; Cold Spring Harbour laboratory Press: Cold Spring Harbour, NY, 2001. 
23. Kropinski, A. M.; Mazzocco, A.; Waddell, T. E.; Lingohr, E.; Johnson, R. P., Enumeration of Bacteriophages by Double Agar Overlay Plaque Assay. In Bacteriophages: Methods and Protocols, Volume 1: Isolation, Characterization, and Interactions, Clokie, M. R. J.; Kropinski, A. M., Eds. Humana Press: Totowa, NJ, 2009; pp 69-76. 
Graphical Abstract

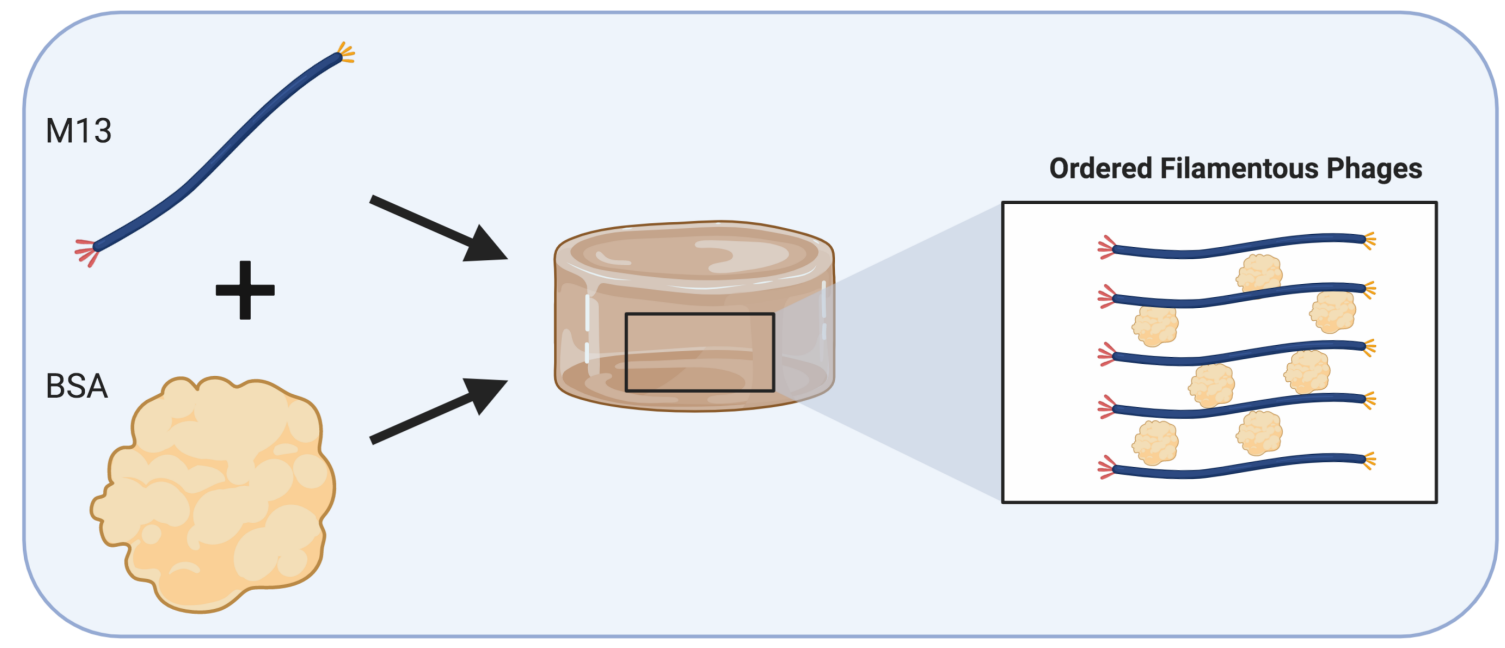

